\title{
Limberg flap as a Primary Treatment for Pilonidal Sinus at Nepal Mediciti Hospital: A Single Unit Experience
}

\author{
Subodh Ghimire', Sunil Kumar Sharma Dhakal ${ }^{2}$, Pranil Rai ${ }^{3}$, Nirvan Rai ${ }^{4}$ \\ ${ }^{3}$ Dr. Pranil Rai, Senior Consultant Surgeon ${ }^{4}$ Dr. Nirvan Rai, Consultant Surgeon \\ Department of General Surgery and Digestive Diseases, Nepal Mediciti Hospital, Lalipur, Nepal
}

'Dr. Subodh Ghimire, Junior Consultant Surgeon ${ }^{2}$ Professor Dr. Sunil Kumar Sharma Dhakal, Principal Consultant Surgeon

\section{ABSTRACT}

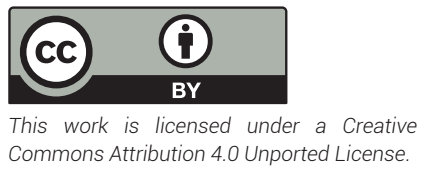

INTRODUCTION Pilonidal Sinus is a common condition with estimated incidence of 260 per million population with more male predilection and is often seen in sacrococcygeal area but has also been described in other areas with hair. The Limberg rhomboid flap was designed by Limberg in 1946 for the closure of a sixty degree rhombus shaped defect with a transposition flap. We would like to share our single unit experience of Rhomboid Limberg flap for Pilonidal sinus in our hospital.

METHODS This is an ongoing prospective descriptive study in the Department of General Surgery and Digestive Diseases of Nepal Mediciti Hospital from October 2018. The demographic of the patients, presenting symptoms, duration of symptoms and previous interventions are recorded and the Rhomboid Limberg flap surgery is done under regional or general anesthesia in prone position. The patient is given intravenous antibiotic and is instructed to sleep laterally or in prone position for 24 hours. The dressing is removed and the wound is inspected for flap status in the next morning. The patient is discharged on 1 st post-operative day on oral antibiotics for 7 days and is followed on 7 th post-operative day for removal of suction drain and alternate sutures in the skin and remaining sutures are removed on 10th post-operative day.

RESULTS We have done 28 cases of Limberg flap for Pilonidal Sinus from October 2018 to December 2020 of which 24 were male patients. None of our patients had flap necrosis. One patient presented with accidental removal of suction drain on 5th postoperative day, however he didn't develop any wound related complication. One patient had superficial wound dehiscence on 10th post-operative day which healed on its own with dressing. One patient developed seroma from 5th postoperative and was managed with dressing and antibiotics and it healed in 2 weeks. None of our patients have developed recurrence and their wounds have healed satisfactorily with minimal scarring and pain. All the patients were able to resume their regular activities within 21 days. All except 2 patients were satisfied with cosmetic outcome.

CONCLUSION The Limberg flap is ideal treatment for pilonidal sinus with minimal postoperative pain, short hospital stay, few complications, rapid return to normal activities, good cosmesis, and a low recurrence rate with short learning curve for young surgeons. Hence, Limberg flap should be routinely used as primary treatment for pilonidal sinus.

KEY WORDS: Limberg flap, Pilonidal Sinus, Primary treatment

\section{INTRODUCTION}

The term Pilonidal was coined by Hodge in 1880 in which pilus means a hair and nidus is a nest.1 Pilonidal Sinus is a common condition with estimated incidence of 260 per million population with more male predilection and is often seen in sacrococcygeal area but has also been described in other areas with hair like axilla, suprapubic, periumbilical and in between the fingers in barbers. ${ }^{2,3}$ The underlying pathophysiological feature is dead hairs being pushed into skin abrasions by movement of the buttocks, which causes a foreign-body reaction within the presacral subcutaneous tissue and development of subsequent acute and chronic abscess and causes significant discomfort to the patient. ${ }^{2,4}$
Incision and drainage is the mainstay of surgical treatment for drainage of pilonidal abscesses in emergency setting but there are number of different surgical treatment options with varying risks and post-operative recurrence rates for elective pilonidal surgery such as excision and laying open, excision and primary closure, incision and marsupialization, advancement flaps like Karydakis procedure, Z flap and $\checkmark-Y$ flap and rotational flaps such as the Limberg flap and also newer and fewer invasive modalities like injection of

*Corresponding Author

Dr. Subodh Ghimire, Junior Consultant Surgeon

Department of General Surgery and Digestive Diseases, Nepal Mediciti Hospital, Bhaisepati, Lalitpur, Nepal

Mobile: +977-9841547225 Email: soobot7@gmail.com 
fibrin glue or thrombin gelatine matrix and endoscopic sinus treatment. ${ }^{5}$

The Limberg rhomboid flap was designed by Limberg in 1946 for the closure of a sixty degree rhombus shaped defect with a transposition flap. ${ }^{6}$ When used for pilonidal sinuses, the technique carries the advantage of excising a rhombus-shaped area of tissue which includes the area of sinus disease and subsequently closing it with a transposition flap of matching shape. ${ }^{5}$ We would like to share our single unit experience of Rhomboid Limberg flap for Pilonidal sinus in our hospital.

\section{METHODS}

This is an ongoing prospective descriptive study in the Department of General Surgery and Digestive Diseases of Nepal Mediciti Hospital from October 2018. We have included all the patients presenting to us with pilonidal sinus in this study. The demographic of the patients, presenting symptoms, duration of symptoms and previous interventions are recorded and the Rhomboid Limberg flap surgery is done under regional or general anesthesia in prone position. The antibiotic prophylaxis is done with 1 grams of ceftriaxone within 60 minutes of the surgery.

The pilonidal region is well shaved and the buttocks are retracted laterally with adhesive tapes. The surgical area is cleaned with $5 \%$ povidone iodine and draped. The opening is identified and $5-10$ milliliters of methylene blue dye is injected. Then a vertical line (AC in Figure 1) about $10 \mathrm{~cm}$ long is drawn in the midline to include all the openings and exactly at the midpoint of that line another line of $6 \mathrm{~cm}$ is drawn which perpendicular to the first line (BD in Figure 1). The ends of the lines are joined to form a rhomboid. From the point $B$ a line equal in length to $A D$ is extended laterally to point $E$ (Figure 1) and another line is drawn parallel to $B C$ and is equal in length to $A B$ to mark for the flap. Then the dissection of the rhomboid encompassing the sinus is done up to the sacral fascia and hemostasis is achieved. The fascio - cutaneous flap is raised in the previously marked area and is rotated to close the defect without tension. The flap is sutured in two layers with Monosyn suture 2.0 RB inside (Figure 2) and Prolene 2.0 CB outside over a negative suction $8 \mathrm{~F}$ mini vacuum drain (Figure 3 ) and a compression dressing is applied. The removed sinus tissue is sent for histopathological examination. The patient is given intravenous antibiotic and is instructed to sleep laterally or in prone position for 24 hours. The dressing is removed and the wound is inspected for flap status in the next morning. The patient is discharged on 1st post-operative day on oral antibiotics for 7 days and is followed on 7th post-operative day for removal of suction drain and alternate sutures in the skin and remaining sutures are removed on 10th post-operative day. The proper management of hair in the pilonidal region is the most important factor for recurrence so the patients are routinely followed in the outpatient clinic every 3 monthly (Figure 4) for a year. The patients are advised for proper hair removal by using depilatory cream or razor.

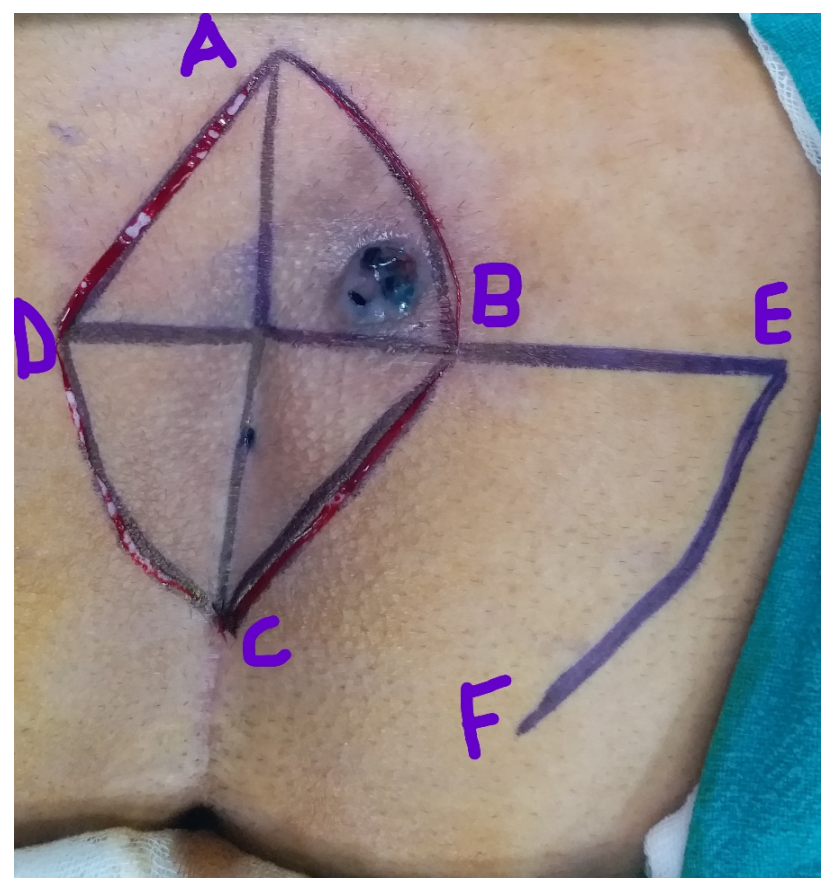

Figure 1 Initial Marking for excision of sinus and flap creation

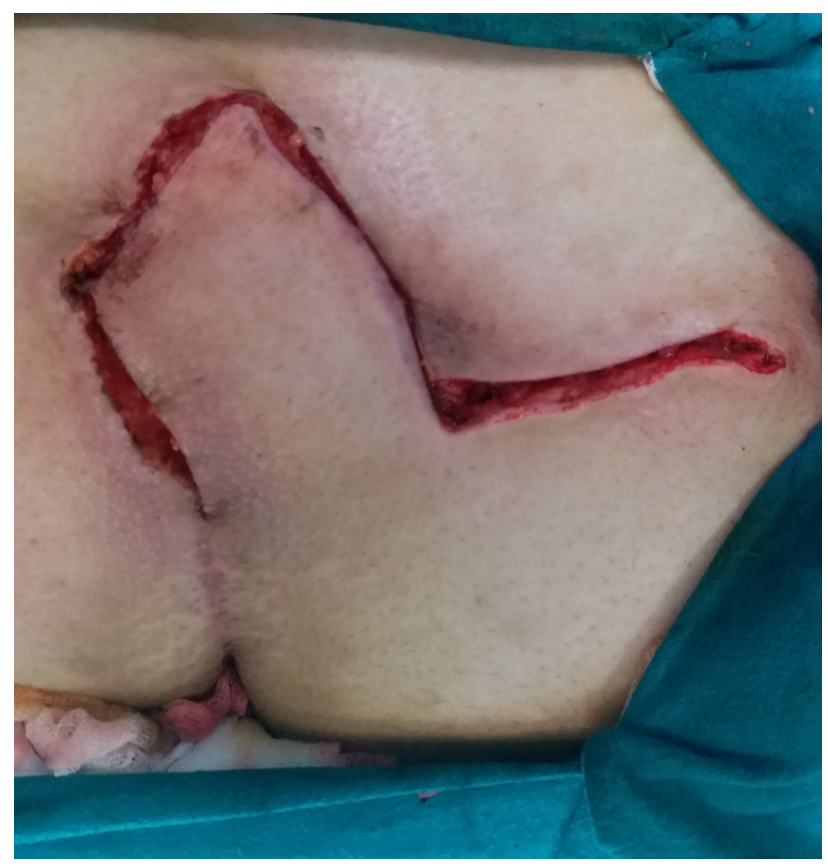

Figure 2 After rotation of flap and closure of inner layer 


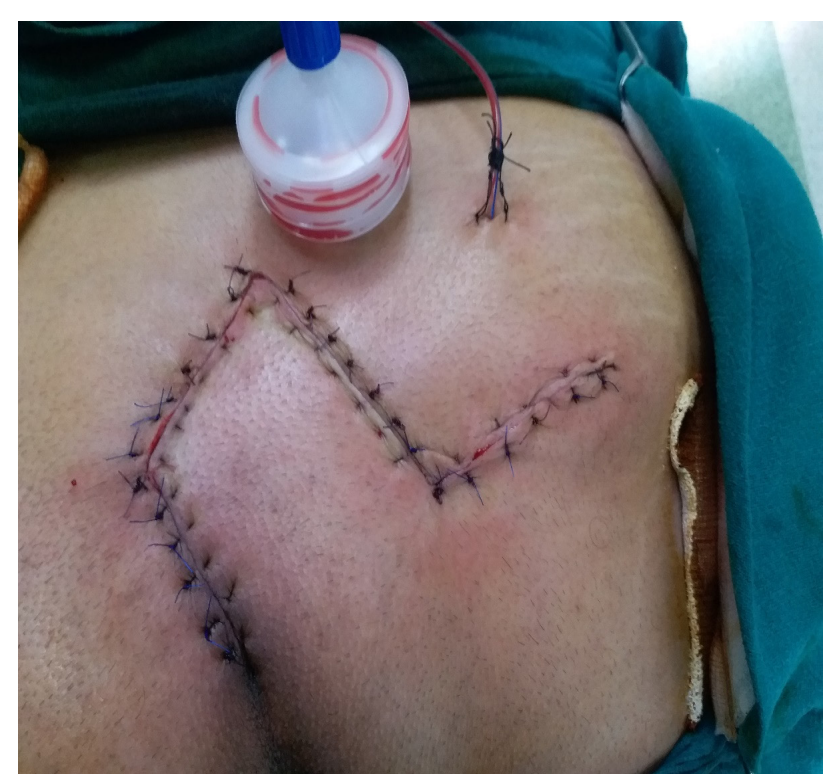

Figure 3 Final appearance

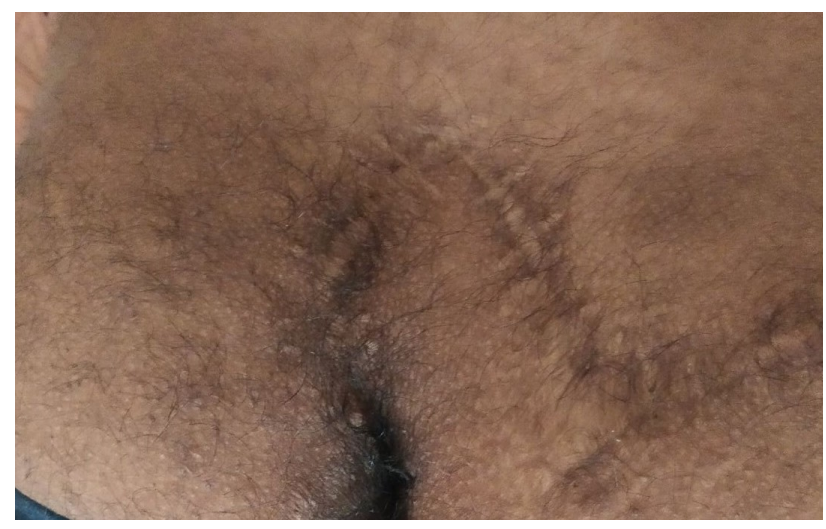

Figure 4 Pilonidal region in six months follow up of one patient

\section{RESULTS}

We have done 28 cases of Limberg flap for Pilonidal Sinus from October 2018 to December 2020 of which 24 were male patients. All except one patient presented electively in the outpatient clinic with pilonidal sinus. One patient presented with abscess which was drained initially and sinus was dealt with later on. Three patients had recurrent pilonidal sinus and had been treated at other center with excision and primary closure. Of the 28 patients 22 had multiple openings in the midline, two had multiple openings in the midline with one opening off midline with granulomatous change communicating with the opening in the midline, rest had single opening. Twenty-seven patients were discharged on first post-operative day, one patient was discharged on 2nd postoperative day on patients' preference.

None of our patients had flap necrosis. One patient presented with accidental removal of suction drain on 5th postoperative day, however he didn't develop any wound related complication. One patient had superficial wound dehiscence on 10th post-operative day which healed on its own with dressing. One patient developed seroma from 5th post-operative and was managed with dressing and antibiotics and it healed in 2 weeks. None of our patients have developed recurrence and their wounds have healed satisfactorily with minimal scarring and pain. All the patients were able to resume their regular activities within 21 days. All except 2 patients were satisfied with cosmetic outcome.

\section{DISCUSSION}

Pilonidal sinus disease is a common condition affecting the natal cleft more commonly than other sites. ${ }^{7}$ The etiopathogenesis of pilonidal disease has been a topic of debate for many years, and theories have moved from the earlier congenital theory to the, now, more accepted acquired theories of Bascom and Karydakis. ${ }^{8-10}$ It has a striking male preponderance with the literature quoting a broad range from 4:1 to 19:1.1,2,11 In our study also we found male preponderance $(85.71 \%)$ for developing pilonidal sinus which also corroborates with findings of Atul S et al. ${ }^{12}$ The mean age of our patients is $24.57 \pm 5.58$ years.

Various surgical techniques have been devised for management of Pilonidal sinus but now the Limberg flap is well accepted all around the globe. There are various studies which have compared the Limberg flap with other various techniques and all the studies found that the Limberg flap is superior to all others. Similarly a meta-analysis comparing primary closure including off-midline techniques with Limberg Flap, showed that the Limberg procedure was associated with less disease recurrence, lower wound infection and dehiscence and they have supported the use of Limberg flap for Pilonidal sinus. ${ }^{13}$

Mentes et al., Can et al., Topgül et al., and Müller et al. have reported complication rate ranging from 3.1\% to $25.7 \%$ after Limberg flap surgery for pilonidal sinus which is comparable with $10.71 \%$ complications in our study. ${ }^{14-17}$ In our study we found none of our patients had flap necrosis, 3.57\% had superficial wound dehiscence, $3.57 \%$ had seroma and none of our patients have recurrence. A systematic review and local experience done by Mohamed $B$ et al. have found the rate of flap necrosis from 0 to $6 \%$, rate of wound dehiscence after Limberg flap from 0 to $25 \%$, rate of seroma from 0 to $9.4 \%$ and recurrence is seen in up to $7.4 \%$ which is comparable with that of our study. ${ }^{5}$ Müller et al. found that cosmetic outcome after Limberg flap was largely accepted by the majority of their seventy patients, likewise nearly all of our patients (92.85\%) are satisfied with the cosmetic outcome. $^{14}$

A study done in Pune, India to study about the learning curve for Pilonidal sinus have concluded that Limberg's flap 
procedure appears to be superior to Karydakis' procedure, and the results are reproducible even by budding surgeons with little experience in the procedure. ${ }^{7}$

\section{CONCLUSION}

The Limberg flap is ideal treatment for pilonidal sinus with minimal postoperative pain, short hospital stay, few complications, rapid return to normal activities, good cosmesis, and a low recurrence rate with short learning curve for young surgeons. Hence, Limberg flap should be routinely used as primary treatment for pilonidal sinus.

\section{REFERENCES}

1. Hodges RM. Pilo-Nidal Sinus. Bost Med Surg J. 1880 Nov 18;103(21):485-6.

2. Søndenaa K, Andersen E, Nesvik I, Søreide JA. Patient characteristics and symptoms in chronic pilonidal sinus disease. Int J Colorectal Dis. 1995 Feb;10(1):39-42.

3. McCallum I, King PM, Bruce J. Healing by primary versus secondary intention after surgical treatment for pilonidal sinus. Cochrane Database of Systematic Reviews. John Wiley and Sons Ltd; 2007.

4. Humphries AE, Duncan JE. Evaluation and Management of Pilonidal Disease. Vol. 90, Surgical Clinics of North America. Surg Clin North Am; 2010. p. 113-24.

5. Boshnaq $M$, Phan $Y C$, Martini I, Harilingam $M$, Akhtar $M$, Tsavellas G. Limberg flap in management of pilonidal sinus disease: systematic review and a local experience. Acta Chir Belg. 2018;118(2):78-84.

6. Wolfe SA. Alexander A. Limberg, M.D. 1894-1974. Plast Reconstr Surg. 1975;56(2):239-40.

7. Gupta A, Anand S, Mehrotra S, Khidtta K. Learning Curve for Pilonidal Sinus Surgery: The Best Option for Budding Surgeons. World J Surg. 2017 Feb 1;41(2):615-9.

8. Kooistra HP. Pilonidal sinuses. Review of the literature and report of three hundred fifty cases. Am J Surg. 1942 Jan 1;55(1):3-17.

9. Bascom J. Pilonidal disease: origin from follicles of hairs and results of follicle removal as treatment. Surgery. 1980;87:56772.

10. Karydakis GE. Easy and Successful Treatment for Pilonidal Sinus after Explanation of its Causative Process. Aust N Z J Surg. 1992;62(5):385-9.

11. Akinci OF, Kurt M, Terzi A, Atak I, Subasi IE, Akbilgic O. Natal cleft deeper in patients with pilonidal sinus: Implications for choice of surgical procedure. Dis Colon Rectum. 2009 May;52(5):1000-2.

12. Sharma DA. Limberg Flap Repair: Our Experience. J Med Sci Clin Res. 2017 Oct 15;5(10).

13. Horwood J, Hanratty D, Chandran P, Billings P. Primary closure or rhomboid excision and Limberg flap for the management of primary sacrococcygeal pilonidal disease? A meta-analysis of randomized controlled trials. Color Dis. 2012 Feb;14(2):14351.

14. Müller K, Marti L, Tarantino I, Jayne DG, Wolff K, Hetzer FH. Prospective Analysis of Cosmesis, Morbidity, and Patient Satisfaction Following Limberg Flap for the Treatment of Sacrococcygeal Pilonidal Sinus. Dis Colon Rectum. 2011 Apr;54(4):487-94.

15. Mentes O, Bagci M, Bilgin T, Ozgul O, Ozdemir M. Limberg flap procedure for pilonidal sinus disease: Results of 353 patients. Langenbeck's Arch Surg. 2008 Mar;393(2):185-9.

16. Topgül K, Özdemir E, Kiliç K, Gökbayir H, Ferahköşe Z. LongTerm Results of Limberg Flap Procedure for Treatment of Pilonidal Sinus: A Report of 200 Cases. Dis Colon Rectum. 2003 Nov;46(11):1545-8.

17. Can MF, Sevinc MM, Hancerliogullari O, Yilmaz M, Yagci G. Multicenter prospective randomized trial comparing modified Limberg flap transposition and Karydakis flap reconstruction in patients with sacrococcygeal pilonidal disease. Am J Surg. 2010 Sep;200(3):318-27. 